\title{
THE IMPACT OF CN LINE PROFILE MEASUREMENTS ON THE COSMIC MICROWAVE BACKGROUND TEMPERATURE
}

\author{
M. E. Kaiser and E. L. Wright \\ University of California, Los Angeles \\ Los Angeles, California USA
}

\begin{abstract}
We present moderate to high signal-to-noise high-resolution $(R \approx 150,000-170,000)$ optical spectra toward $\zeta$ Oph. Gaussian fits to our data indicate a value of the line-width parameter $b$, of $b=1.4 \pm 0.2 \mathrm{~km} \mathrm{~s}^{-1}$, along this line of sight. When $\mathrm{CN}$ is used as an indirect probe of the cosmic microwave background (CMB) temperature, the line profile is used to determine saturation corrections in the line. This affects column density calculations, which are reflected in the excitation temperature. Current measurements of the $b$-value along this line of sight range from $0.88 \pm 0.02 \mathrm{~km} \mathrm{~s}^{-1}$ (Crane et al. 1986) to $1.3 \pm 0.1 \mathrm{~km} \mathrm{~s}^{-1}$ (Hegyi, Traub, and Carleton 1972). The extreme range of these $b$-values yield saturation corrections to the CMB temperature that differ by 0.05 $\mathrm{K}$, which is equal to the quoted precision of current measurements. Preliminary analysis of observations toward HD 29647 indicate that $T_{C M B}=2.70 \pm 0.14 \mathrm{~K}$ at $2.64 \mathrm{~mm}$ toward this line of sight.
\end{abstract}

The CN radical in interstellar space is excited primarily by the CMB radiation field at $2.64 \mathrm{~mm}$ and $1.32 \mathrm{~mm}$. Optical spectroscopy is used to determine the column densities and consequently the excitation temperature $T_{e x}$ of the $J=1-0$ and $J=2-1$ rotational transitions. In the absence of local excitation, $T_{C M B}=T_{e x}$.

Unfortunately, these optical lines are narrow and easily saturated. To determine the saturation corrections, it is necessary to resolve the line profile or do a curve-of-growth analysis. We believe the former is preferable, especially when multiple clouds contribute to the absorption. In this case, the line profile cannot be described by a simple model such as a Gaussian. The curve-of-growth method, which depends upon the intrinsic line profile, now requires observations of a large number of lines arising from a common ground state. For $\mathrm{CN}$, it is difficult to obtain observations of enough lines to conduct such a multi-cloud analysis. Assuming a single cloud for this case would overestimate the line-width parameter and underestimate the saturation corrections for either a curve-of-growth analysis or a Gaussian fit to the line profile. When the line can be described by a Gaussian, the saturation corrections to the column density depend upon $b$.

High resolution measurements of the $C N B^{2} \Sigma-X^{2} \Sigma(0,0)$ transition family toward $\zeta$ Oph have also been performed by Hegyi, Traub, and Carleton (1972), who measured $b=1.30 \pm 0.1 \mathrm{~km} \mathrm{~s}^{-1}$ with a resolution of $R=300,000$, and Crane et al. (1986), who determined that $b=0.88 \pm 0.02 \mathrm{~km} \mathrm{~s}^{-1}$ with a resolution of $R \approx 150,000$. Our results, $b=1.4 \pm 0.2 \mathrm{~km} \mathrm{~s}^{-1}$, agree with the Fabry Perot results of Hegyi, Traub, and Carleton (1972). However, since our method is similar to that of Crane et al. (1986), we are concerned about the discrepancy between our results and have tried to find an explanation.

High-resolution radio measurements of $\mathrm{CO}$ toward $\zeta$ Oph (Langer, Glassgold, and Wilson 1987) show a minimum of four clouds. If this cloud structure is also exhibited by $\mathrm{CN}$, then our Gaussian fits to the observed optical line profile overestimate the line-width parameter. We have attempted three types of fits to determine how best to model the cloud structure and subsequently determine the column densities. First, we independently fit the calibration line and the $R(0)$ line with single Gaussians. The resulting line-width parameters were subtracted in 
quadrature to obtain $b=1.4 \pm 0.2 \mathrm{~km} \mathrm{~s}^{-1}$. Second, for a data subset, we fit our calibration spectra with a two-Gaussian model. Convolving this spectrograph response with a single Gaussian fit to the $\mathrm{R}(0)$ line, we obtain $b=1.3 \pm 0.1 \mathrm{~km} \mathrm{~s}^{-1}$. While the $\chi^{2}$ of these two fits is similar, the residuals from the first fit show a systematic pattern due to a non-Gaussian red tail in our line profiles. Finally, we abandoned the Gaussian assumption. We generated a model based on Langer, Glassgold, and Wilson's multi-cloud CO results, convolved it directly with our calibration lamp spectra, and then fit the data. We believe this is the correct approach. However, before we can quote column densities or an excitation temperature for this line of sight, we must explore further the parameter space associated with this model.

Determinations of $T_{C M B}$ from $\mathrm{CN}$ measurements have improved with the proliferation of CCD detectors and high-resolution spectrographs, so a careful search for systematic errors is essential. Our concern has been with the effect of saturation corrections. In general, the $R(0)$ line $(J=0-1$ transition) is saturated and the $R(2)$ line is virtually undetectable for the historically popular lines of sight. Therefore lines of sight with smaller column densities of $\mathrm{CN}$ may provide more accurate determinations of $\mathrm{CN}$ at $2.64 \mathrm{~mm}$, whereas new lines of sight with larger CN column densities are certainly required for a precise measurement at $1.32 \mathrm{~mm}$.

We also present preliminary lower-resolution results toward HD 29647. Our line profiles are unresolved. The narrow $B^{2} \Sigma-X^{2} \Sigma C N$ lines are saturated, necessitating a curve-of-growth analysis. Consequently, we observed the near-infrared $A^{2} \Pi-X^{2} \Sigma(1,0)$ and $(2,0)$ bands toward this line of sight. Unfortunately, they reside within a forest of telluric features that obscure many of the lines. Our preliminary reduction of these observations yields an excitation temperature of $T_{e x}=2.78 \pm 0.14 \mathrm{~K}$. Crutcher (1986) has observed this line at $113 \mathrm{GHz}$ $(2.64 \mathrm{~mm})$ with the Kitt Peak $11 \mathrm{~m}$ radio telescope obtaining a local excitation temperature of $T_{\text {loc }}=0.08 \mathrm{~K}$. Correcting our excitation temperature for local excitation yields $T_{C M B}=2.70 \pm 0.14 \mathrm{~K}$ at $2.64 \mathrm{~mm}$ along this line of sight.

Acknowledgments-This work was supported in part by NASA contract NAS527670 to UCLA. Observations were conducted at Lick Observatory, University of California.

\section{REFERENCES}

Crane, P., Hegyi, D. J., Mandolesi, N., and Danks, A. C. 1986, Ap. J., 309, 822.

Crutcher, R. M. 1985, Ap. J., 288, 604.

Hegyi, D. J., Traub, W. A., and Carleton, N. P. 1972, Phys. Rev. Letters, 28, 1541.

Langer, W. D., Glassgold, A. E., and Wilson, R. W. 1987, Ap. J., 322, 450.

Meyer, D. M., and Jura, M. 1985, Ap. J., 297, 119.

Thaddeus, P. 1972, ARAA, 10, 305. 